\title{
Heterobostrychus hamatipennis Lesne, 1895, un ravageur mineur importé de Chine, nouveau pour la faune de France (Coleoptera, Bostrichidae)
}

\author{
par Henri-Pierre ABERLENC
}

CIRAD-BIOS, UMR 55 CBGP, TA A-55/L, F - 34398 Montpellier cedex 5 <henri-pierre.aberlenc@cirad.fr>

Résumé. - Heterobostrychus hamatipennis Lesne, 1895 (Coleoptera Bostrichidae) est cité de France pour la première fois, dans des meubles importés de Chine en 2003 et en 2007. Son statut de ravageur, sa biologie, sa répartition orientale originelle et son expansion à travers le monde à la faveur de la croissance des exportations chinoises sont discutés.

Summary. - Heterobostrychus hamatipennis Lesne, 1895, a minor pest recently imported from China for the first time into France (Coleoptera, Bostrichidae). Heterobostrychus hamatipennis Lesne, 1895 (Coleoptera Bostrichidae) is recorded from France for the first time in furniture imported from China in 2003 and 2007. Its pest status, biology, primitive oriental distribution and its expansion throughout the world connected with the increased in chinese exportations are discussed.

Keywords. - Coleoptera, Bostrichidae, invasive species from China, xylophage, orientale, australian and holarctic regions.

\section{ARRIVÉE EN FRANCE D’UN NOUVEAU RAVAGEUR}

En juillet 2003, une entreprise française qui importe des meubles fabriqués en Chine me confiait un couple d'un Bostryche indéterminé, que je pus identifier grâce à l'excellent travail de LESNE (1898). C'était la première fois à ma connaissance qu' Heterobostrychus hamatipennis Lesne, 1895, était découvert dans notre pays, et même en Europe ! En septembre 2008, la même entreprise m'a envoyé un individu mâle de la même espèce (émergence le 4 juillet 2008) et un échantillon du meuble attaqué (fabriqué au cours du second semestre 2007), une porte constituée d'une âme en Pin (Pinus sp.), avec les extrémités et un placage vernis en Paulownia tomentosa. La galerie est creusée dans l'âme en Pin et le trou de sortie circulaire mesure $5 \mathrm{~mm}$ de diamètre.

\section{RÉPARTITION ORIGINELLE}

A l'époque de la publication de LESNE (1898), sa répartition géographique connue était limitée à la région orientale (Inde, Bouthan, Sri Lanka, Chine méridionale, Viêt-Nam, Laos, Japon, Philippines, Malaisie et Indonésie: Bornéo) et à la région afrotropicale, sous-région malgache (Madagascar et île Maurice). Le peuplement humain de Madagascar s'est produit pendant plusieurs siècles voici environ deux millénaires par des vagues successives de migrants venus d'Indonésie, qui arrivaient dans des bateaux capables de transporter jusqu'à mille personnes et plusieurs centaines de tonnes de fret. On peut donc raisonnablement supposer qu'ils ont importé Heterobostrychus hamatipennis avec eux, dans du mobilier, cette espèce étant très vraisemblablement originaire de la région orientale.

\section{EXPANSION MONDIALE $\left(\mathrm{XX}^{\mathrm{e}}\right.$ ET $\mathrm{XXI}^{\mathrm{e}}$ SIÈCLE)}

Région néarctique: USA (Caroline du Nord et Floride, avant 1988); Canada, en 19971998, dans du bois et du mobilier importé d'Asie.

Région paléarctique : Belgique, en juillet 2005, dans des objets en osier importés de Chine (Quingdao) en octobre 2004 (FASSOTTE, 2005); Corée, en 2005, dans des grumes (CHOO et al., 1983); Espagne, en septembre 2007, dans un échantillon dégradé de fibres de Palmier. 

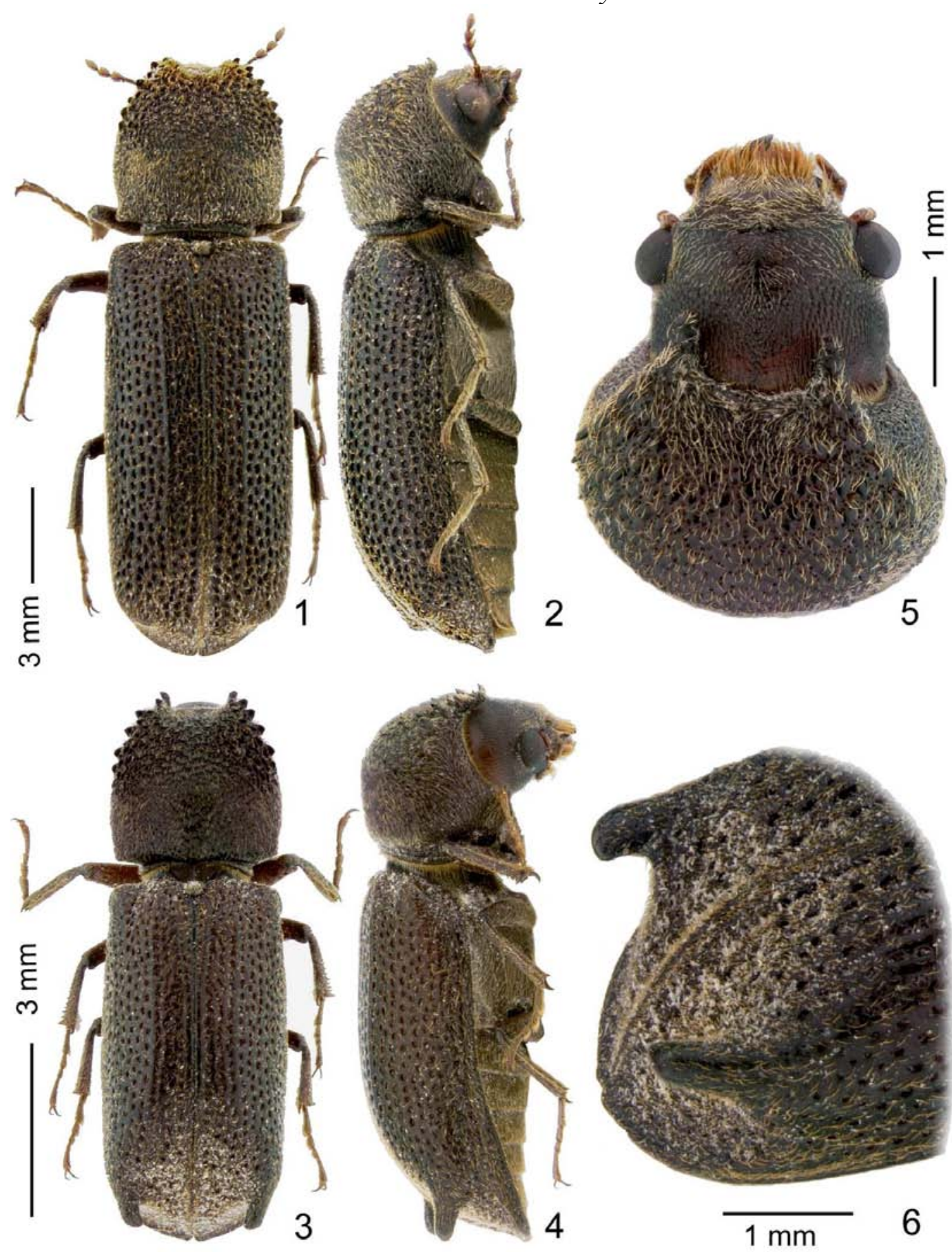

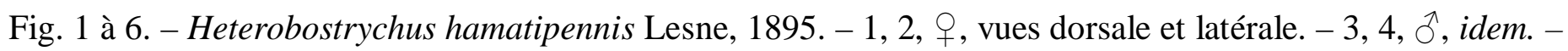
5, $\widehat{o}$, tête et pronotum. -6 , $\widehat{O}$, déclivité de l'apex élytral. (phot. H.-P. Aberlenc)

Régions australienne et pacifique: Hawaii [Oahu], en juin 2003, dans une branche morte de Manguier; Australie, en avril 2007, dans du Saule importé de Chine.

On peut s'attendre à de nombreuses citations nouvelles.

\section{BIOLOGIE}

Heterobostrychus hamatipennis est cité du Kapokier rouge Bombax malabaricum (Malvaceae), du Saule Salix sp. (Salicaceae), de divers Bambous (Poaceae Bambousoideae), du Canarium pimela (Burseraceae), du Manguier Mangifera indica (Anacardiaceae), du Pin Pinus sp. (Pinaceae), d'un Palmier (Arecaceae), etc. : il est polyphage. L'espèce s'attaque aux arbres morts comme au bois sec utilisé en menuiserie (bois de calage et d'emballage, meubles, objets divers) et en vannerie (osier). On peut le déceler grâce aux bruits ténus, grattements dus aux mandibules des larves creusant leurs galeries, et à la sciure suspecte en résultant; le trou d'émergence circulaire mesure de 4 à $6,5 \mathrm{~mm}$ de diamètre. Selon BEESON (1937), le cycle est annuel avec des émergences en juin-juillet dans l'hémisphère nord. 


\section{STATUT DE RAVAGEUR}

En France, trois individus ont été signalés en cinq ans par une entreprise qui importe régulièrement du mobilier made in China: le taux d'attaque est très faible. Il est hautement vraisemblable que d'autres introductions non publiées se produisent depuis plusieurs années, à la faveur des importations chinoises, mais rien ne suggère que l'espèce puisse s'être acclimatée en France.

Dans le monde, son expansion ne s'est produite qu'au cours du XX $\mathrm{X}^{\mathrm{e}}$ siècle et elle continue aujourd'hui. Il semble que l'espèce ne s'implante pas durablement dans les pays où elle est signalée. Il est plutôt rassurant de ne trouver que des cas isolés dans le flux considérable de marchandises provenant de Chine: sauf changement brutal de son éthologie, cette espèce n'occasionne que des dommages limités. En Chine, c'est un ravageur mineur qui ne fait pas assez de dégâts pour mobiliser les chercheurs. On peut donc rester raisonnablement optimiste, les attaques devraient rester exceptionnelles, les dégâts ponctuels, jamais massifs ni durables : il peut donc être considéré comme un ravageur d'importance secondaire, mais aucune mauvaise surprise ne peut être exclue à l'avenir et il est bon de rester vigilant.

\section{CARACTÈRES UTILES POUR LA DÉTERMINATION}

Imago : 8-15 mm. Brun foncé. Dessus du corps couvert d'une pubescence jaune, courte, éparse et couchée. Mandibules à apex pointus, se croisant. Front avec une ligne médiane longitudinale, clypéus avec une carène médiane longitudinale, suture fronto-clypéale nette (caractères plus aisés à voir chez le mâle). Bord antérieur du pronotum concave, dépourvu de dents, la zone antérieure médiane du pronotum étant légèrement excavée. Angles antérieurs du pronotum du mâle formant chacun une apophyse saillante dentée (fig. 5). Tarses aussi longs que les tibias. Ponctuation élytrale faite de gros points enfoncés à bords nets, plus petits vers l'apex. Déclivité apicale des élytres du mâle plus densément pubescente que le reste de la surface élytrale, avec de chaque côté une apophyse marginale dont la pointe apicale émoussée est tournée vers l'intérieur (fig. 6). Déclivité apicale des élytres de la femelle avec un calus de chaque côté.

REMERCIEMENTS. - Je remercie MM. Patrick Vernange et Laurent Vasseur pour m'avoir confié leurs échantillons à identifier, le Dr Mic Julien du CSIRO pour l'amélioration du summary, ainsi que mon collègue et ami Laurent Soldati dans le laboratoire duquel j'ai pu utiliser l'équipement photo multifocus Entovision®.

\section{AUTEURS CITÉS}

BEESON G.F.C., 1937. - The Ecology and Control of the Forest Insects of India. Shiva Offset Press, Dehra Dun India.

CHOO H.Y., WOO K.S., LEE C.K., 1983. - Classification of the Bostrichidae intercepted from imported timbers II. Korean Journal of Plant Protection, 22 (1): 30-33.

FASSOTTE C., 2005. - Première observation en Belgique et en Europe de l'espèce exotique Heterobostrychus hamatipennis (Lesne, 1895) (Coleoptera Bostrychidae) sur de l'osier. Département "Lutte biologique et Ressources phytogénétiques" du Centre wallon de Recherches Agronomiques, Gembloux, Belgique.

LESNE P., 1898. - Révision des Coléoptères de la famille des Bostrychides. $3^{\mathrm{e}}$ Mémoire. Bostrychinae. Annales de la Société entomologique de France, 67 : 438-621.

WALKER K., 2008. - Chinese auger beetle (Heterobostrychus hamatipennis). Pest and Diseases Image Library. Téléchargeable en ligne: http://www.padil.gov.au (consulté le 28 octobre 2008) 